DOI: https://doi.org/10.15688/mpcm.jvolsu.2020.4.2

UDC 517.442

Submitted: 15.07 .2020

LBC 511

Accepted: 06.11.2020

\title{
REGULARITY OF THE TRANSFORM OF LAPLACE AND THE TRANSFOM OF FOURIER
}

\author{
Andrey V. Pavlov \\ Candidate of Physical and Mathematical Sciences, Associate Professor, \\ Department of Higher Mathematics-1, \\ MIREA - Russian Technological University \\ a_pavlov@mirea.ru \\ Prosp. Vernadskogo, 78, 119454 Moscow, Russian Federation
}

\begin{abstract}
Regularity of the transform of Laplace in the opened area of 0 is proved with the help of some methods of the transform of Fourier. The class of the transform of Laplace from the transform of Fourier is considered from some functions without a regularity in null. The functions are regular in the opened area of 0 . It is proved that the sine transform of Fourier from the cosine transform of Fourier is equal to the cosine transform from the sine transform of Fourier on the module.
\end{abstract}

Key words: transform of Fourier, transform of Laplace, regularity of the double transform of Laplace, transposition of the sine and cosine transforms of Fourier.

\section{Introduction}

In article the methods of complex analysis are applied to research of the transform of Fourier. The main part of the work is considered in the remark 1 and the proposition 2 . As a result we obtain a regularity of the double transform of Laplace in some open area of null $[3 ; 4 ; 6 ; 7]$ (the remark 1 ); by definition, the $f(z)$ function is regular in the $z$ point, if in the \& point exists the $d f(z) / d z$ derivative. In the [7] work from the fact is proved, that the sine \& transform is equal to the cosine transform of Fourier on the module (in the article the fact $>$ is not proved fully). The methods of research of the article were first considered in the [3;4] $\varangle$ work of author. The proposition 1 in the non-obvious form was first considered in the [4] article. From the propositions we obtain, that the sine transform of Fourier from the cosine transform of Fourier is equal to the cosine transform from the sine transform of Fourier on (2) the module (theorem 1). 
By definition, the $L Z(t)(\cdot)(x)$ function is the transform of Laplace

$$
L Z(t)(\cdot)(x)=\int_{0}^{\infty} e^{-x t} Z(t) d t, x \in[0, \infty),
$$

and

$$
\begin{gathered}
L_{ \pm} Z(t)(\cdot)(x)=\int_{0}^{\infty} e^{ \pm x t} Z(t) d t, x \in[0, \infty), L_{-}=L, \\
F_{ \pm} u(t)(\cdot)(p)=\int_{-\infty}^{\infty} e^{ \pm p i t} u(t) d t, p \in(-\infty, \infty), \\
C^{0} u(t)(\cdot)(x)=\int_{0}^{\infty} u(t) \cos x t d t, \quad S^{0} u(t)(\cdot)(x)=\int_{0}^{\infty} u(t) \sin x t d t, x \in(-\infty, \infty), \\
F_{ \pm}^{0} u(t)(\cdot)(p)=\int_{0}^{\infty} e^{ \pm p i t} u(t) d t, p \in(-\infty, \infty) .
\end{gathered}
$$

\section{Regularity of the transform of Laplace and the transform of Fourier}

In the section we use the $\mathrm{Y} 1$ and $\mathrm{R} 1$ conditions.

The Y1 condition takes place for the $u(p)$ function, if the $u(p)$ function is regular in $p: p \in G_{\varepsilon}=\{|\operatorname{Re} p|<\varepsilon \bigcup|\operatorname{Im} p|<\varepsilon\}$ for some $\varepsilon>0, u(0)=0$, and

$$
\max \left[|u(p)|,|d u(p) / d p|,\left|d^{2} u(p) / p^{2}\right|\right]\left|p^{2+\delta}\right| \rightarrow 0,|p| \rightarrow \infty
$$

$\delta>0, \delta=$ const., Re $u(x)=u(x), x \in(-\infty, \infty)$.

The $\mathbf{R} \mathbf{1}$ condition takes place for the $l_{1}(p), L_{1}(p), l_{2}(p), L_{2}(p)$ functions, if the $l_{1}(p), L_{1}(p)$ functions are regular in left part of the complex plane for all $R e \leq 0$, the $l_{2}(p), L_{2}(p)$ functions are regular in right part of the plane for all $R e \geq 0$, if the $l_{1}(p), L_{1}(p), l_{2}(p), L_{2}(p)$ functions are continuous for all $p \in(-i \infty, i \infty)$ from the side of definition of the functions, and the $l_{1}(p), L_{1}(p), l_{2}(p), L_{2}(p)$ functions are limited in the area of the definition (with the values on the $(-i \infty, i \infty)$ boundary):

$$
\begin{gathered}
\sup _{R e \geq 0} l_{2}(p) \leq c_{0}, \sup _{R e \geq 0} L_{2}(p) \leq c_{0} ; \\
\sup _{R e \leq 0} l_{1}(p) \leq c_{0}, \sup _{R e \leq 0} L_{1}(p) \leq c_{0}, c_{0}=\text { const }, c_{0}<\infty .
\end{gathered}
$$

\section{Proposition 1.}

$$
l_{1}(p)-L_{1}(p)=L_{2}(p)-l_{2}(p) \equiv 0, p=i t, t \in(-\infty, \infty),
$$

if the $R 1$ condition takes place for the $l_{1}(p), L_{1}(p), l_{2}(p), L_{2}(p)$ functions, and

$$
l_{1}(p)+l_{2}(p)=L_{1}(p)+L_{2}(p), p=i t, t \in(-\infty, \infty) .
$$


Proof. We use $l_{1}(p)-L_{1}(p)=L_{2}(p)-l_{2}(p), p=i t, t \in(-\infty, \infty)$. From the $\mathrm{R} 1$ condition the $L_{2}(p)-l_{2}(p)$ function is an analytical continuation of the $l_{1}(p)-L_{1}(p)$ function from the left to the right part of the plane across the complex axis [2]. The functions are limited in area of its definition and on the complex axis $(-i \infty, i \infty)$ (by the $\mathrm{R} 1$ condition). The function is regular in the full complex plane and is limited in the plane. We obtain $l_{1}(p)-L_{1}(p)=L_{2}(p)-l_{2}(p) \equiv$ const $=0$. [?; ?].

The proposition 1 is proved.

In the lemma 1 and the theorem 1 we consider the $l_{1}(p), L_{1}(p), l_{2}(p), L_{2}(p)$ functions:

$$
\begin{gathered}
l_{1}(p)=L_{+} F_{-} u(t)(\cdot)(p), \operatorname{Re} p<0 ; l_{2}(p)=L F_{+} u(t)(\cdot)(p), \operatorname{Re} p>0, \\
L_{1}(p)=L_{+} F_{-} u_{-}(t)(\cdot)(p), \operatorname{Re} p<0 ; L_{2}(p)=L F_{+} u_{-}(t)(\cdot)(p), \operatorname{Re} p>0,
\end{gathered}
$$

where ,by definition,

$$
u_{-}(t)=u(t), t \in[0,+\infty), u_{-}(t)=-u(t), t \in(-\infty, 0),
$$

and for the $u(p)$ function the Y1 condition takes place.

Lemma 1. 1) For the $l_{1}(p), L_{1}(p), l_{2}(p), L_{2}(p)$ functions the $R 1$ condition takes place, if for the $u(p)$ function the Y1 condition takes place.

2) $l_{1}(p)+l_{2}(p)=L_{1}(p)+L_{2}(p)=2 \pi u(p / i), p \in G_{\varepsilon}$, if for the $u(p)$ function the $Y 1$ condition takes place.

Proof. The $l_{1}(p), L_{1}(p), L_{2}(p), l_{2}(p)$ functions (in the form of the Laplace transform) are limited in the area of its definition and on the complex axis $(-i \infty, i \infty)$ (the fact is wellknown $[3 ; 4 ; 6 ; 7]$, if $u(0)=0$, we use the Y1 condition [?;?] with help of the formula of integration by parts in the inlying integral for the $l_{1}(p)=L_{+} F_{-} u(t)(\cdot)(p), L_{1}(p)=$ $=L_{+} F_{-} u_{-}(t)(\cdot)(p), l_{2}(p), L_{2}(p)$ functions [8]).

From the same formula of integration by parts we obtain, that the $l_{1}(p), L_{1}(p), L_{2}(p)$, $l_{2}(p)$ functions are continuous for all $p \in(-i \infty, i \infty)$ from the side of definition of the functions, if $u(0)=0$.

It is obvious, the $l_{1}(p), L_{1}(p), L_{2}(p), l_{2}(p)$ functions are regular in the area of its definition without the $(-i \infty, i \infty)$ boundary (in the points exists the $d f(p) / d p$ derivative, if for the $u(p)$ function the Y1 condition takes place [8]).

The first part of the lemma 1 is proved.

From $l_{1}(i y)=\overline{l_{2}(i y)}, L_{1}(i y)=\overline{L_{2}(i y)}, y \in[0,+\infty)$, we obtain

$$
2 \operatorname{Re}_{1}(i y)=l_{1}(i y)+l_{2}(i y), L_{1}(i y)+L_{2}(i y)=2 \operatorname{Re} L_{1}(i y), p=i y,
$$

$y \in[0,+\infty)$. We can use the inverse operator of the transform of Fourier [1], and $2 \operatorname{Re} l_{1}(i y)=2 \pi u(y), 2 \operatorname{Re} L_{1}(i y)=2 \pi u_{-}(y), y \in[0,+\infty)$, where $u(y)=u_{-}(y), y \in$ $\in[0,+\infty)$. We get

$$
l_{1}(i y)+l_{2}(i y)=L_{1}(i y)+L_{2}(i y)=2 \pi u(y), y \in[0,+\infty) .
$$

It is well-known, that the $l_{1}(p), l_{2}(p)$ functions are regular for all $p: p \in\{|\operatorname{Re} p|<$ $<A \cup|\operatorname{Im} p|<A>0\}$, if the Y1 condition for the $u(p)$ function takes place for some $\varepsilon>A>0[2-4]$. 
We can use, that from the $\mathrm{R} 1$ condition the $l_{1}(p), L_{1}(p), l_{2}(p), L_{2}(p)$ functions are continuous for all $p \in(-i \infty, i \infty)$ from the side of definition of the functions .

We get $L_{1}(p)=2 \pi u(p / i)-L_{2}(p), p \in G_{\varepsilon}$ as the analytical continuation of the $L_{1}(p)$ functions [2]) across the $[0,+\infty)$ axis, and

$$
l_{1}(p)+l_{2}(p)=L_{1}(p)+L_{2}(p)=2 \pi u(p / i), p \in G_{A}, A<\varepsilon,
$$

[2] (the equality takes place in the area of regularity of the $u(z)$ function too, $z=p / i$ ).

The second part of the lemma 1 is proved.

Theorem 1. $\left.S^{0} C^{0} u(t)(\cdot)(x)+C^{0} S^{0} u(t)(\cdot)(x)\right) \equiv 0, x \in[0,+\infty)$, if for the $u(p)$ function the Y1 condition takes place, and $\operatorname{Re} u(t)=u(t), t \in[0, \infty)$.

Proof. To use the proposition 1 we mast prove, that $l_{1}(i y)+l_{2}(i y)=L_{1}(i y)+L_{2}(i y), y \in$ $\in(-i \infty, i \infty)$.

From the lemma 1 we get the equality for $y \in[0, i \infty)$.

For $y \in(-i \infty, 0)$ we will prove the remark 1 .

Remark 1. The $L L u(x)(\cdot)(z)$ function is regular in $\{z:-A<\operatorname{Re} z<A\}$ for some $A>0$, if for the $u(p)$ function the Y1 condition takes place with $\varepsilon>A$ (we obtain, that the $\operatorname{LLu}(x)(\cdot)(z)$ function is non multiple-meaning function in $\{z:-A<\operatorname{Im} z<A\}[2 ; 4])$.

Proof. The $L L(z)$ function is regular in $R e w>0$, if $L L(w)=L L u(x)(\cdot)(z)$. The fact we get from the $L L u(x)(\cdot)(z)=\int_{0}^{\infty}[u(x) /(z+x)] d x$ equality after a change of the limits of integration in the $L L u(x)(\cdot)(z)$ function, $R e z>0$ [8] (the derivative of $L L(z)$ is defined in $R e z>0$ in the Y1 condition [2]).

The main part of the remark 1 is the proposition 2 .

In formulation of proposition 2 the new definitions are used: the reflection of the $U(p)$ function in relation to the $(A, 0)$ point as the $U_{A \rightarrow}(p)$ function, $U(p)=U(p-A+$ $+A), U_{A \rightarrow}(p)=U(-(p-A)+A)=U(2 A-p)((0,0)$ is the center of co-ordinates); the $2 \mathrm{~A}$-moving of the $\mathrm{U}(\mathrm{p})$ function is the $U(p-2 A)$ function. It is obviously, if we will move the $U(p)$ function to the right on $2 A$ value we obtain the $U(p-2 A)$ function.

Proposition 2. 1) $U(p-2 A)=U_{A \rightarrow}(p),|p-2 A|<A>0$ if and only if $U(-p)=U(p)$, $|p|<A>0$, if the $U(p)$ function is defined in $|p|<A$.

2) The $V(p)=Y(p),|p-2 A|<A>0$ equality takes place, where the $V(p)=U_{A \rightarrow}(p)$ function is the result of reflection of the $U(p)$ function in relation to the $(A, 0)$ point, and the $Y(p)$ function is the result of reflection of the $U(p-2 A)$ function in relation to the $(2 A, 0)$ point (the $(0,0)$ point is the center of co-ordinates, and the $U(p)$ function is defined in $|p|<A)$. The $U(A-z)=U_{A \rightarrow}(p)$ equality takes place for the $(A, 0)$ center of co-ordinates, $z=p-A, \operatorname{Re} p>A-a>0, a \in(-\infty, \infty)$, if the $U(p)$ function is defined in Rep $>A+a$ (for all $A>0)$.

Proof. The first part of proposition we obtain from $U(2 A-p)=U_{A \rightarrow}(p)$, where $U(2 A-$ $-p)=U(p-2 A)$, if and only if $U(z)=U(-z)((0,0)$ is the center of coordinates).

The $U_{A \rightarrow}(p)=U(2 A-p)=V(p)$ equality takes place as the definition of the $U_{A \rightarrow}(p)$ reflection $((0,0)$ is the center of co-ordinates). The $2 \mathrm{~A}$-moving of the $\mathrm{U}(\mathrm{p})$ function is the 
$U(p-2 A)$ function; the result of the reflection of the $U(p-2 A)$ function in relation to the $(2 A, 0)$ point is the $Y(p)$ function:

$$
Y(p)=U((4 A-p)-2 A)=U(2 A-p)=V(p) .
$$

The second part of proposition we obtain from the $Y(p)=V(p)$ equality for the new center of co-ordinates in the $(A, 0)$ point. In the $U(-(z-A))=U_{A \rightarrow}(p)$ equality $U_{A \rightarrow}(p)=U(-z)$ , $z=p-A, \operatorname{Re} z>0$, if we consider the $U(-z), U(-(z-A))$ function as the comparison of points of the complex plane to the points on other plane (with $z=p-A$ ).

The proposition 2 is proved.

With help of the proposition 2 from $U(-(z-A))=U_{A \rightarrow}(p)=U(-z)$ with $U(-z)=$ $=L L(z)$ we obtain, that the $L L(z)$ is regular in $\operatorname{Re} z>-A<0$, if the $U(-z)$ function is regular for all $z: \operatorname{Re} z>0$ [2] (the $U(z)$ function from the second part of the proposition 2 is defined for all $\operatorname{Re} p>A+a, a=A$ ).

The remark 1 is proved.

From the second part of the lemma 1 we obtain $L_{1}(i y)+L_{2}(i y)=2 \pi u(y), y \in[0, i \infty)$.

From the remark 1 the $L_{1}(p)$ function is regular in $p: p \in\{-A<\operatorname{Re} p<A>0\}$ (we use, that the $L_{1}(p)$ function is equals to the sum of the two values of the double transform of Laplace $[1 ; 8])$.

We get $L_{1}(p)+L_{2}(p)=2 \pi u(p / i), p \in G_{A}$ for some $A>0$ [2], and $L_{1}(i y)+L_{2}(i y)=$ $=2 \pi u(y), y \in(-i \infty, 0)$.

It is well-known, that the $l_{1}(p), l_{2}(p)$ functions from the lemma 1 are regular for all $p: p \in\{|\operatorname{Re} p|<A \bigcup|\operatorname{Im} p|<A>0\}$, if the $u(p)$ function is regular in $p: p \in\{|\operatorname{Re} p|<$ $<\varepsilon \bigcup|\operatorname{Im} p|<\varepsilon\}$ for some $\varepsilon>A>0[2-4]$.

The $l_{1}(i y)+l_{2}(i y)=L_{1}(i y)+L_{2}(i y), y \in(-i \infty, i \infty)$, is proved.

We can use the proposition 1 , and

$$
l_{1}(p)-L_{1}(p)=L_{2}(p)-l_{2}(p) \equiv 0 .
$$

(All other conditions of the proposition 1 are the result of the first part of the lemma 1).

The result of the theorem 1 we obtain from

$$
\begin{gathered}
0 \equiv l_{2}(p)-L_{2}(p)=2 \int_{0}^{\infty} e^{-p t} d t \int_{-\infty}^{0} e^{i t x} u(x) d x= \\
\left.=2 \int_{0}^{\infty} e^{-p t} d t \int_{0}^{\infty} e^{-i t x} u(-x) d x=-i\left[S^{0} C^{0} u(-t)(\cdot)(x)+C^{0} S^{0} u(-t)(\cdot)(x)\right)\right], \\
p=i x, x \in[0,+\infty), \text { (with } u(-t)=u(t), t \in[0, \infty))[2 ; 3 ; 5-8] .
\end{gathered}
$$

\section{Conclusion}

Results of the theorem 1 and proposition 2 probably are of interest for the further study from point of physical applications. 


\section{REFERENCES}

1. Kolmogorov A.N., Fomin S.V. Elements of the Theory of Functions and Functional Analysis. Moscow, Nauka Publ, 1976. 544 p.

2. Lavrentiev M.A., Shabat B.V. The Methods of Theory Functions of Complex Variable. Moscow, Nauka Publ, 1987. 688 p.

3. Pavlov A.V. About the Equality of the Transform of Laplace to the Transform of Fourier. Issues of Analysis, 2016, vol. 23, no. 1, pp. 21-30.

4. Pavlov A.V. The Fourier Transform and New Inversion Formula of the Laplace Transform. Math. notes, 2011, vol. 90, no. 6, pp. 793-796.

5. Pavlov A.V. The New Inversion of Laplace Transform. Journal of Mathem. and Syst. Scien., 2014, vol. 4, no. 3, pp. 197-201.

6. Pavlov A.V. The Regularity of the Laplace Transform. Math. Phys.and Comp. Model., 2019, vol. 22, no. 1, pp. 5-11.

7. Pavlov A.V. Permutability of Cosine and Sine Fourier Transforms. Journal Moscow University Mathematics Bulletin, 2019, vol. 74, no. 2, pp. 75-78.

8. Fihtengoltz G.M. The Course of Differential and Integral Calculus, vol. II. Moscow, Nauka Publ, 1969. 800 p.

\section{РЕГУЛЯРНОСТЬ ПРЕОБРАЗОВАНИЯ ЛАПЛАСА И ПРЕОБРАЗОВАНИЕ ФУРЬЕ}

\section{Андрей Валерианович Павлов}

Кандидат физико-математических наук, доцент кафедры высшей математики-1, МИРЭА - Российский технологический университет

a_pavlov@mirea.ru

просп. Вернадского, 78, 119454 г. Москва, Российская Федерация

Аннотация. Доказана регулярность двойного преобразования Лапласа в окрестности нуля. Рассматривается класс функций с нарушением регулярности в нуле. Преобразование Лапласа от преобразования Фурье от функций из данного класса регулярно в окрестности нуля. Доказана перестановочность синус и косинус преобразований Фурье в условиях регулярности двойного преобразования Лапласа в окрестности нуля.

Ключевые слова: преобразование Фурье, преобразование Лапласа, регулярность двойного преобразования Лапласа, перестановочность синус и косинус преобразований Фурье. 